\title{
A Rare Case of Decapsulation Injury in a Transplant Kidney: A Case Study and Review of the Literature
}

\author{
Ashwini Paranjpe $\mathrm{MD}^{1^{*}}$, Namita Alkokar $\mathrm{MD}^{2}$, Sam Sultan $\mathrm{MD}^{3}$, Stephen Stampp $\mathrm{MD}^{4}$, Erica \\ Riddick ${ }^{1}$, Jim Kim MD ${ }^{5}$ and Anthony Watkins MD $^{5 *}$ \\ ${ }^{1}$ Department of surgery, Lokmanya Tilak Municipal General Hospital, Mumbai, India \\ ${ }^{2}$ Department of Surgery, Howard University Hospital, Washington, DC, USA \\ ${ }^{3}$ Department of Surgery, University of Maryland School of Medicine, Baltimore, Maryland, USA \\ ${ }^{4}$ MountainView Regional Medical Center, Las Cruces, New Mexico, USA \\ ${ }^{5}$ Department of Surgery, NewYork-Presbyterian, Weill Cornell Medical College, New York, USA
}

*Corresponding Authors: Anthony Watkins, Department of Surgery, NewYork-Presbyterian, Weill Cornell Medical College, New York, USA, Tel: 2127465330; Fax: 2127468720: E-mail: acw9003@ med.cornell.edu

Ashwini Paranjpe, Department of surgery, Lokmanya Tilak Municipal General Hospital, Mumbai, India, E-Mail: ashwiniparanjpe14@gmail.com

Received: 09 November 2017; Accepted: 07 December 2017; Published: 14 December 2017

\begin{abstract}
Postoperative perinephric collections are known complications of a transplant kidney with decapsulation injury being one of its rare etiologies. It can be early onset due to capsular injury during laparoscopic donor nephrectomy or late onset usually due to immunosuppressive medications or spontaneous in nature. We report a rare case of late onset decapsulation injury following insertion of a percutaneous nephrostomy drain for treating hydronephrosis. It was subsequently treated with marsupialization of the collection and creating a peritoneal window. Complete resolution of the perinephric collections was noted on follow up. On review literature, it was found that there is no unanimous management protocol related to decapsulation injuries. We have made an attempt to concise various treatment approaches mentioned in the literature for such cases and their outcomes. In case of patients with late onset decapsulation injuries such as ours, marsupialization has shown success in majority of cases obviating the need for nephrectomy. We suggest that marsupialization can form a reliable first line treatment following late onset decapsulation without any clear etiology, in majority of cases.
\end{abstract}


Keywords: Perinephric collection; Decapsulation; Marsupialization; Percutaneous Nephrostomy

\section{Introduction}

Despite many refinements in the operative approach, complications continue to account for a significant cause of morbidity following renal transplantation. Postoperative perinephric collections are one of the relatively common findings with reported incidence ranging from 20-50\%. These collections are typically due to seromas, lymphoceles, urinomas, and hematomas that can result in a mass effect on adjacent structures leading to pain, swelling and allograft dysfunction [1-4]. One rare cause of perinephric collections is decapsulation injury that can be the result of capsular injury during laparoscopic donor nephrectomy or immunosuppression medications. The classification of these collections has been controversial. Herein, we report an unusual case of decapsulation in a transplanted kidney following placement of a percutaneous nephrostomy ( $\mathrm{PCN}$ ) tube for suspected obstructive uropathy more than two decades following renal transplantation. To our knowledge, this is the latest reported onset of decapsulation injury with an unique etiology.

\section{Case Report}

The patient is a 55-year-old man with history of end-stage renal disease (ESRD) of unclear etiology who received a deceased donor renal transplant in 1991. The patient had an uncomplicated postoperative course and had not experienced any significant transplant related issues such as rejection episodes or major infections in the ensuing years. He was in his usual state of health when he presented to the outpatient clinic in August 2014 with acute kidney injury (AKI) with a rise in serum creatinine from a baseline of $1.0 \mathrm{mg} / \mathrm{dL}$ to $2.5 \mathrm{mg} / \mathrm{dL}$. Workup included an ultrasound of the renal allograft, which revealed new hydronephrosis that was thought to be the etiology of the AKI (Figure 1).

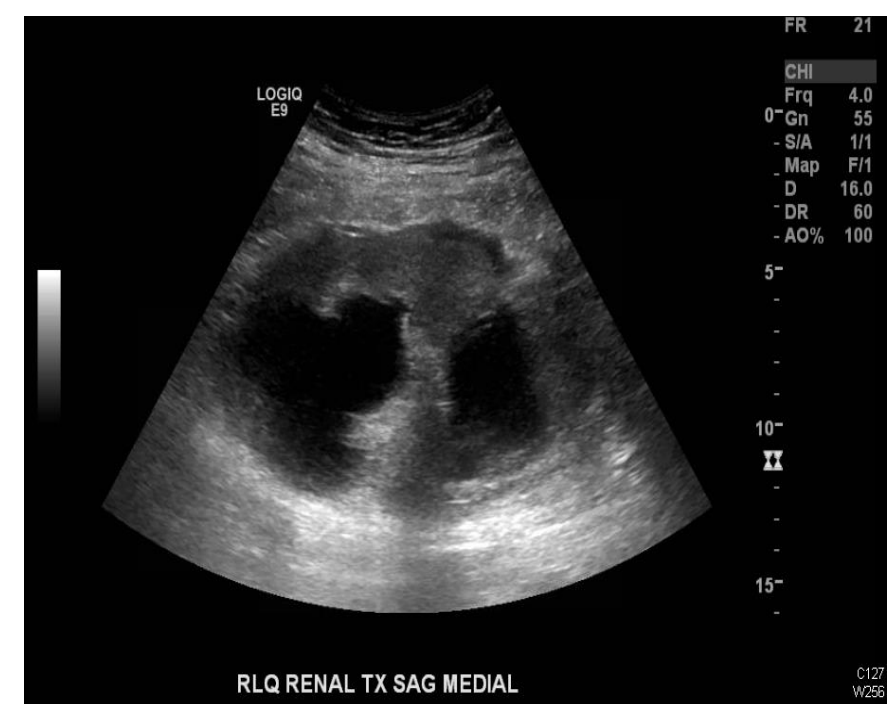

Figure 1: Etiology of the AKI.

The patient underwent placement of a percutaneous nephrostomy (PCN) tube by the interventional radiology (IR) service (Figure 2). Despite adequate placement and drainage, the creatinine did not improve over the following days. A week later the patient presented to the emergency room with fever, nausea, vomiting, diffuse abdominal pain and 
decreased oral intake. The creatinine level remained elevated $(3.0 \mathrm{mg} / \mathrm{dL})$ and a repeat ultrasound and CT demonstrated resolution of the hydronephrosis, however a new subcapsular complex collection measuring $13.0 \times 2.6$ $\times 8.6 \mathrm{~cm}$ and a contiguous lower pole perinephric collection measuring $8.3 \times 4.4 \times 4.6 \mathrm{~cm}$ were visualized (Figures 3 and 4). The patient was admitted to the hospital for further work up and IR placement of a perinephric drain into the subcapsular collection (Figure 5). In addition, a Pseudomonas urinary tract infection was appropriately treated with Piperacillin/Tazobactam. A cell count with differential was performed on this fluid and the creatinine level was $2.0 \mathrm{mg} / \mathrm{dL}$ (Table 1).

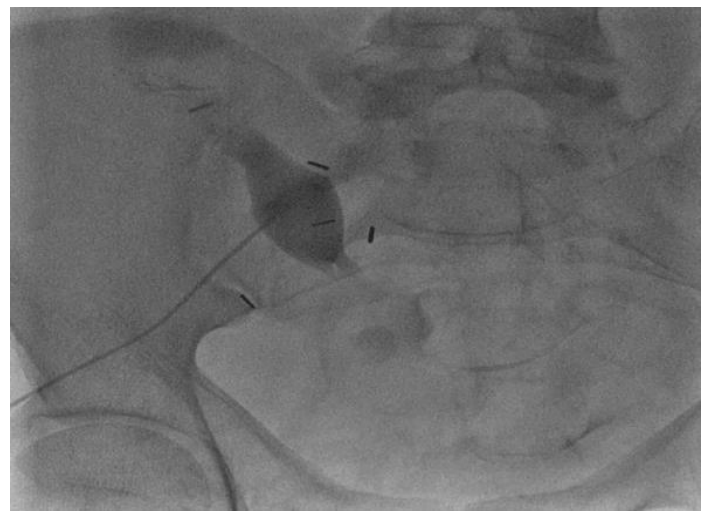

Figure 2: Placement of a percutaneous nephrostomy (PCN) tube.

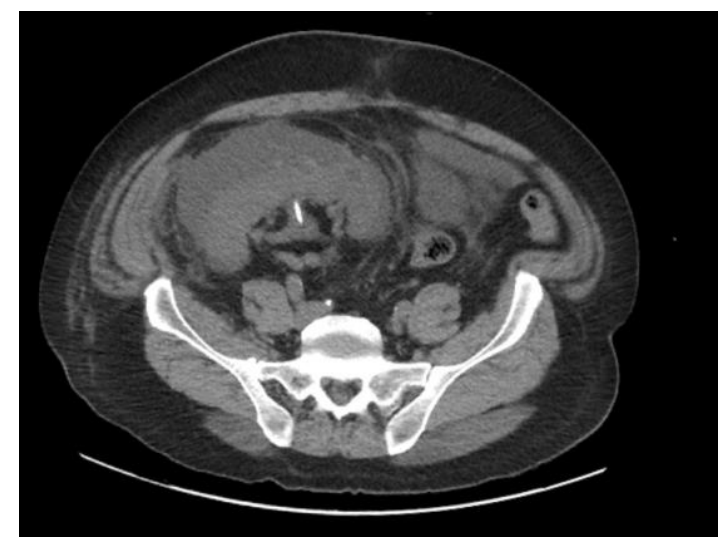

Figure 3: Subcapsular complex collection measuring.

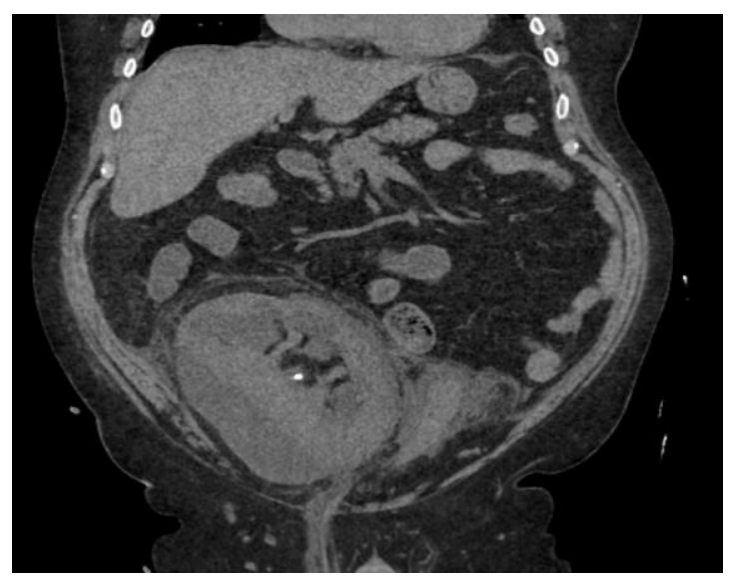

Figure 4: Contiguous lower pole perinephric collection measuring. 


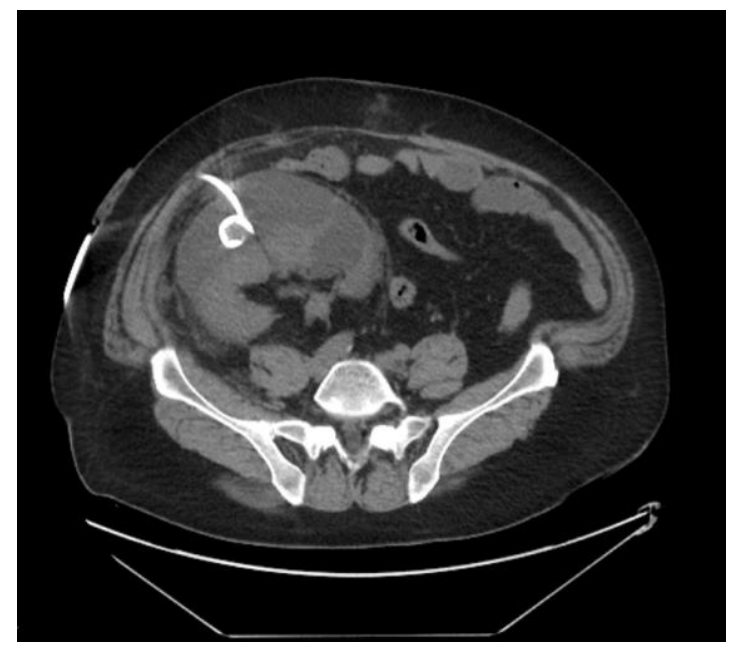

Figure 5: IR placement of a perinephric drain into the subcapsular collection.

\begin{tabular}{|l|l|l|}
\hline Test & Result & Unit \\
\hline Color & Pink & - \\
\hline Appear & Cloudy & - \\
\hline RBC BF & 3650 & $/ \mathrm{ul}$ \\
\hline WBC BF & 160 & $/ \mathrm{ul}$ \\
\hline Segs BF & 19 & $\%$ \\
\hline Lymph BF & 41 & $\%$ \\
\hline Mono/Macrophage BF & 40 & $\%$ \\
\hline
\end{tabular}

Table 1: Differential count of body fluid.

The subcapsular drain had persistent high output (450-750cc/day), therefore a nuclear medicine lasix renogram was also performed that was negative. A tentative diagnosis of a lymphocele was made and an attempt at sclerosis with daily instillation of betadine was performed. The patient developed pain over the graft site. Repeat imaging revealed a persistent subcapsular collection. The decision was made to take the patient to the operating room for exploration and marsupialization of the lymphocele. Intraoperatively, it was noted that the allograft kidney had become decapsulated and a peritoneal window was created to allow communication into the peritoneal cavity (Figure 6). An intraoperative biopsy revealed active and chronic tubulointerstitial inflammation, mild interstitial fibrosis/tubular atrophy (IFTA), diffuse diabetic glomerulosclerosis and arteriosclerosis, and extensive arteriolar intimal hyalinosis with obliterative changes. These findings were consistent with the recent hydronephrosis and recurrent diabetic nephropathy, however there was no definite evidence of cell mediated or antibody mediated rejection. One week following the procedure, follow up imaging confirmed resolution of the perinephric collection with residual hydronephrosis (Figure 7). Of note, his creatinine improved to a new baseline of $2.0 \mathrm{mg} / \mathrm{dL}$. 


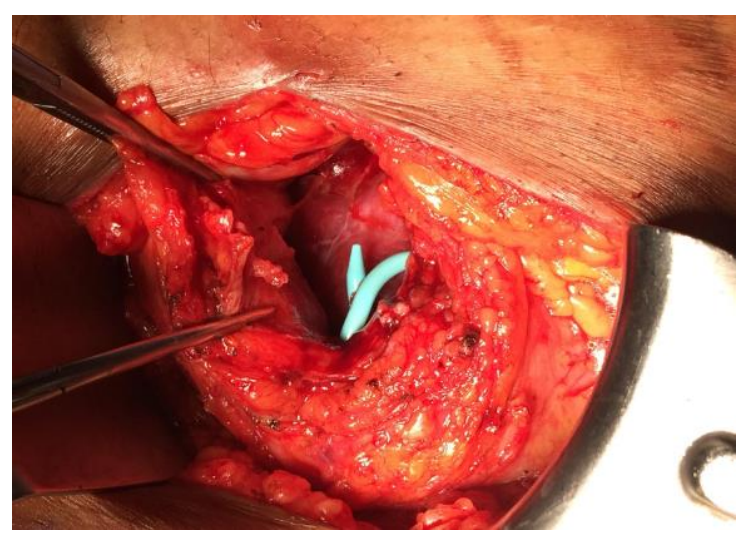

Figure 6: Peritoneal window to allow communication into the peritoneal cavity.

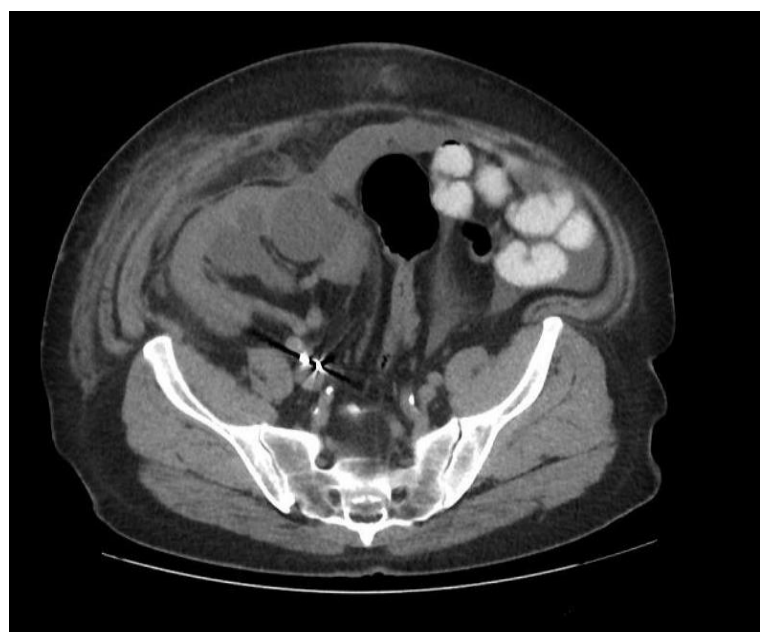

Figure 7: Confirmed resolution of the perinephric collection with residual hydronephrosis.

\section{Discussion}

Decapsulation injuries of the renal allograft have been noted to occur following renal transplantation and are typically divided between early and late presentations. Early cases have typically been related to injury resulting from the donor nephrectomy. For example, Mohamed et al. [5] reported a case of decapsulation at the time of delivery of the kidney during laparoscopic donor nephrectomy caused by the endocatch device. The kidney was repaired by suturing the defect and used immediately without any sequelae. Yashwant et al. [6] reported a case of a 37 year old male who underwent living donor renal transplantation that resulted in decapsulation injury which was successfully treated with sclerosis using povidone iodine instillation. In this case, the decapsulation injury occurred secondary to cortical scars and dense adhesions encountered during the open donor nephrectomy. Guleria et al. [7] successfully treated 12 patients that had confirmed lymphatic leaks with $20 \mathrm{~mL}$ of alcohol based 5\% povidone iodine instillation followed by clamping the drain for $20 \mathrm{mins}$ and reopening it and repeating the procedure till there was no further drainage.

Late presentations are typically either spontaneous or related to immunosuppression medications, especially in patients on sirolimus. Yang et al. [8] reported late spontaneous kidney graft decapsulation after conversion to sirolimus for maintenance therapy 11 years post-transplant due to presence of chronic allograft glomerulopathy in a 
recipient. Approximately 9 months later the patient presented with severe pain and swelling over the allograft related to decapsulation injury with associated fluid collection and marsupialization was performed for management. Unfortunately the kidney function continued to deteriorate and the patient was eventually started on hemodialysis followed by transplant nephrectomy. It was possible that sirolimus precipitated the spontaneous decapsulation as lymphocele formation is one of the known side effects. Leal et al. [9] reported the successful treatment of a case of late spontaneous decapsulation by converting the patient from sirolimus to cyclosporine and performing marsupialization.

Late spontaneous decapsulation of a kidney graft with excessive fluid leakage is a rare condition with very few cases being reported in the literature. Herbert et al. [10] using an experimental model of decapsulation had demonstrated that fluid production rises when ureteral pressure or renal venous pressure is increased. Koene et al. [11] described two patients with spontaneous decapsulation occurring late after transplant resulting in the accumulation of large amounts of fluid. Both patients failed multiple attempts to control the accumulation including re-fixating the capsule with butylcyanoacrylate, wrapping the kidney with lyophilized human dura, and the placement of a LeVeen shunt. Both patients ultimately required a nephrectomy for definitive treatment. The authors argue that the source of the fluid probably originated from the renal interstitial tissue in these cases and not lymphatics due to the large quantity of fluid (up to $5 \mathrm{~L} / \mathrm{d}$ ) and low protein levels, including a normal lymphangiogram in one patient as there was no obstruction in the ureter or renal vein. The cause of the decapsulation remains obscure. Renal vein thrombosis or rejection can lead to interstitial edema in the kidney, but these conditions were excluded. They assumed that the edema was secondary to the decapsulation.

Nghiem et al. [12] were among the first to perform marsupialization to successfully treat a subcapsular collection in a patient that developed a lymphocele after transplant . Mestres et al. [13] also successfully performed laparoscopic marsupialization on a patient who had received a cadaveric kidney transplant that failed 12 years later. Myhre et al. [14] also successfully treated spontaneous decapsulation of the kidney 18 years after post transplant by making a 3 inch window in the anterior/cranial wall of the cyst. Others have utilized alternative approaches to treat these complications that have been more successful. Tiggeler et al. [15] successfully treated a patient with excessive fluid leakage after spontaneous decapsulation of a renal allograft 6 years post-transplant with infrared contact coagulation. He then covered the kidney with a slip of omentum which led to decreased fluid leakage with a known follow up of 9 months. Brown et al. [16] obtained a progressive reduction of the collection volume by using furosemide and amiloride (both diuretics) with no recurrence for 6 years in a women five years post-transplant opting to attempt medical management rather than surgical intervention. Mousson et al. [17] reported 2 cases, one 4 years posttransplant and the other 5 years post- transplant with swelling over graft site developing weeks following a 2 meter fall, with successful treatment in both cases. In the first case marsupialization, was carried out to allow for drainage of the collection into the peritoneum with no recurrence after 11 years. In the second case, 4 percutaneous drainage procedures were done that finally led to resolution of the collection. Mekeel et al. [18] wrapped the kidney in cellulose membrane and sealed it with fibrin glue to decrease the fluid excretion in conjunction with marsupialization. In their case the postoperative fluid collection and wound drainage from this patient was unconcentrated urine from the kidney parenchyma. As for most urine leaks, where peritoneal window is not the standard of care and may promote leakage and based on success reported with use of both cellulose membrane and 
fibrin sealant, gelatin matrix thrombin, bovine serum albumin, cyanoacrylate glue, or collagen matrix, they choose to use fibrin glue which worked. On thorough review of literature, we found that our case is unique in terms of the etiology of the decapsulation. Patient developing decapsulation due to a suspected trauma following PCN insertion has not been reported before.

The pathophysiology of late spontaneous decapsulation remains unclear. Because the number of cases reported in the literature is small, there is no unanimity on the management. Management varied and was customised depending on each clinical scenario. The decision to treat, or not to treat as well as how to treat depends on the patient clinical presentation and success has been obtained by different treatments. In our case when fluid re-accumulation occurred following the capping trial, the patient was returned to the operating room was treated by the marsupialization technique and the patient benefitted from the treatment.

\section{Conclusion}

Perinephric collection is a known complication in a transplant kidney. Etiology and its management varies depending upon the timing of its presentation. Decapsulation injury to the kidney is one of the rare causes of perinephric collections. Our patient is a case of late onset decapsulation injury leading to perinephric collections with an unique etiology. Inspite of the distinctive characteristics of such perinephric collections, marsupialization has shown success in majority of cases and it eliminates the need for nephrectomy. Marsupialization can form the first line treatment following late onset decapsulation without any clear etiology in majority of cases.

\section{References}

1. Yap R, Madrazo B, Oh HK, et al. Perirenal fluid collection after renal transplant. Am Surg 47 (1981): 287 290.

2. Haddad MC, Hawary MM, Khoury NJ, et al. Radiology of perinephric fluid collections. Clin Radiol 57 (2002): 339-346.

3. Richard HM. Perirenal transplant fluid collections. Semin Intervent Radiol 21 (2004): 235-237.

4. Ebadzadeh MR, Tavakkoli M. Lymphocele after kidney transplantation: where are we standing now? Urol J 5 (2008):144-148.

5. Mohamed HK, Lin A, Savage SJ, et al. Parenchymal transection of the kidney inflicted by endocatch bag entrapment during a laparoscopic donor nephrectomy. Am J Transplant 6 (2006): 232-235.

6. Yashwant R, Guleria S, Khattar N, et al. Leak from the surface of a decapsulated renal allograft: urine or lymph? Excellent response to povidone iodine instillation. Saudi J Kidney Dis Transpl 25 (2014): 105-108.

7. Guleria S, Mehta SN, Mandal S, et al. Povidone-iodine in the treatment of lymphatic fistulae in renal transplant recipients. Transplant Proc 35 (2003): 327-328.

8. Yang $\mathrm{MH}$, Loong $\mathrm{CC}, \mathrm{Wu} \mathrm{CW}$, et al. Late spontaneous kidney graft decapsulation after administration of sirolimus in a recipient with chronic hepatitis B and C infection: a case report. Transplant Proc 40 (2008): 2437-2439.

9. Leal R, Pinto H, Alves R, et al. Late spontaneous decapsulation of the kidney graft in a patient with hepatitis $\mathrm{C}$ and treated with sirolimus: possible pathophysiological association in a rare complication. BMJ Case Rep (2017). 
10. Hebert LA, Stuart KA, Stemper JA. Effect of renal decapsulation on renal function. Am J Physiol 229 (1975): 632-639.

11. Koene RA, Skotnicki SH, Debruyne FM. Spontaneous renal decapsulation with excessive fluid leakage after transplantation. N Engl J Med 300 (1979): 1030-1031.

12. Nghiem DD, Schulak JA, Corry RJ. Decapsulation of the renal transplant as a mechanism of lymphocele formation. Transplant Proc 14 (1982): 741-742.

13. Mestres J, Sebastià C, Musquera M, et al. Late subcapsular lymphocele in a kidney graft. Transplantation 93 (2012): e35-7.

14. Myhre H-A, Øyen O, Kårbø $\varnothing$, et al. Successful treatment of spontaneous kidney graft decapsulation 18 years after renal transplantation: a case report. NDT Plus 3 (2010): 100-102.

15. Tiggeler RG, van der Sluis RF, Wobbes T, et al. Successful treatment with infrared contact coagulation of excessive fluid leakage after spontaneous decapsulation of a renal allograft. Transplantation 41 (1986): 264-265.

16. Brown AL, Greenwood RN, Cattell WR. Successful medical management of presumed spontaneous decapsulation of a transplant kidney. Nephrol Dial Transplant 5 (1990): 75-76.

17. Mousson C, Zanetta G, Tanter Y, et al. Late "spontaneous" kidney graft decapsulation with fluid collection: lymphocele or transudate? Nephrol Dial Transplant 13 (1998): 1288-1291.

18. Mekeel KL, Mazur MJ, Reddy KS, et al. Diffuse Parenchymal Urine Leak After Kidney Transplantation Following Degloving Injury During Donor Nephrectomy. American journal of transplantation: official journal of the American Society of Transplantation and the American Society of Transplant Surgeons 7 (2007): 2039-2041.

\footnotetext{
(C) This article is an open access article distributed under the terms and conditions of the
Creative Commons Attribution (CC-BY) license 4.0
} 